\title{
Enhanced human bone marrow mesenchymal stem cell functions on cathodic arc plasma- treated titanium
}

This article was published in the following Dove Press journal:

International Journal of Nanomedicine

10 December 2015

Number of times this article has been viewed

Wei Zhu'

George Teel'

Christopher M O'Brien'

Taisen Zhuang'

Michael Keidar'

Lijie Grace Zhang ${ }^{1-3}$

'Department of Mechanical and Aerospace Engineering, ${ }^{2}$ Department of Biomedical Engineering,

${ }^{3}$ Department of Medicine, The George Washington University, Washington, DC, USA
Correspondence: Lijie Grace Zhang Department of Mechanical and Aerospace Engineering, The George Washington University, Science and Engineering Hall, Room 3590, 800 22nd Street Northwest, Washington, DC 20052, USA

Tel + I 2029942479

Fax + I 2029940238

Email lgzhang@gwu.edu
Abstract: Surface modification of titanium for use in orthopedics has been explored for years; however, an ideal method of integrating titanium with native bone is still required to this day. Since human bone cells directly interact with nanostructured extracellular matrices, one of the most promising methods of improving titanium's osseointegration involves inducing biomimetic nanotopography to enhance cell-implant interaction. In this regard, we explored an approach to functionalize the surface of titanium by depositing a thin film of textured titanium nanoparticles via a cathodic arc discharge plasma. The aim is to improve human bone marrow mesenchymal stem cell (MSC) attachment and differentiation and to reduce deleterious effects of more complex surface modification methods. Surface functionalization was analyzed by scanning electron microscopy, atomic force microscopy, contact angle testing, and specific protein adsorption. Scanning electron microscopy and atomic force microscopy examination demonstrate the deposition of titanium nanoparticles and the surface roughness change after coating. The specific fibronectin adsorption was enhanced on the modified titanium surface that associates with the improved hydrophilicity. MSC adhesion and proliferation were significantly promoted on the nanocoated surface. More importantly, compared to bare titanium, greater production of total protein, deposition of calcium mineral, and synthesis of alkaline phosphatase were observed from MSCs on nanocoated titanium after 21 days. The method described herein presents a promising alternative method for inducing more cell favorable nanosurface for improved orthopedic applications.

Keywords: titanium, mesenchymal stem cell, plasma, nanocoating, orthopedics

\section{Introduction}

Although orthopedic implants have become safer and more successful in recent years, it is important to note that current orthopedic implant technology has not been perfected. Considering the endurance of current orthopedic implants (10-15 years), younger and more active patients (men younger than 60 years and women younger than 55 years) will inevitably need more than one revision surgery in their lives. ${ }^{1}$ In the United States by 2030 , it was estimated that the demand for primary total hip and knee replacements is estimated to grow by $174 \%$ to 572,000 and $673 \%$ to 3.48 million procedures, respectively. ${ }^{2}$ Nearly $17 \%$ of hip replacements and $8 \%$ of knee replacements will go on to need revision procedures of previously failed hip and knee replacements. ${ }^{3}$ Due to the strikingly increasing number of patients who need various medical implants and the relatively high percentage of revision procedures performed around the world, it is highly desirable to significantly lengthen the service lifetime of current orthopedic implants and, thus, dramatically reduce patient pain and health insurance costs. 
Generally, one crucial criterion for the long-term success of orthopedic implants is the formation of sufficient bonding between the implant and the juxtaposed bone, which is a process known as osseointegration. ${ }^{4}$ Insufficient bonding to the juxtaposed bone may lead to a mismatch of mechanical properties between implant materials and the surrounding bone tissues, which result in implant loosening. Traditional orthopedic implant materials, such as titanium (Ti), have been chosen based on their excellent mechanical strength, bioinert character, and relative ease of manufacturing, ${ }^{5}$ but there has not been enough consideration about their chemical structure and surface properties for optimal osseointegration. Consequently, bare $\mathrm{Ti}$ is not often well integrated into natural bone, and repeated loading leads to implant loosening in situ. ${ }^{6,7}$ This loosening can become very severe with time and leads to complete failure of the implant, inducing high medical, mental, and quality of life costs. ${ }^{7}$ Thus, many researchers seek to improve the material and chemical properties of Ti to delay or mitigate implant loosening and improve osseointegration in situ.

Human bone has a highly ordered extracellular matrix that consists of numerous nano- to microconstituents, which closely affects various bone marrow mesenchymal stem cells (MSCs), osteoblast (bone-forming cell), and osteoclast (bone-resorbing cell) functions. ${ }^{8,9}$ One approach to improve the osseointegration efficacy of $\mathrm{Ti}$ is to create biomimetic nano- and microsurface texturization of the material of interest. ${ }^{4,10-12}$ With this in mind, numerous surface treatment processes, including anodization, nanomaterial coating, machining or micromachining, particle blasting, and chemical etching, have been developed to modify Ti surface topography. ${ }^{1,8,13,14}$ For example, one of the common methods to induce roughness to $\mathrm{Ti}$ implants is to use a combination of sandblasting and hot sulfuric/hydrochloric acid etchants, a process known as sand-blast, acid-etch, for producing a highly texturized surface morphology. ${ }^{15-17}$ Ti modified in this way exhibits a host of improvements markedly in bone differentiation; however, the necessary corrosive acid mixture required for effective etching of Ti implants impose some potential risks on employing this technique. Although modifying the surface of $\mathrm{Ti}$ is not uncommon, many of these processes can be complex or tend to be time and labor intensive. Sand-blast, acid-etch-treated implants, for example, go through a multistep process that utilizes two acid washes, sandblasting, and chemical modification under nitrogen for each implant. ${ }^{18}$ In addition, $\mathrm{Chu}^{19}$ and Chu et al ${ }^{20}$ did a lot of work about plasma-surface modification. In one of their studies, they modified Ti using calcium plasma to improve the bioactivity. ${ }^{21}$ When the modified implant is exposed to air, the calcium reacted with water to generate calcium hydroxide and then reacted with carbon dioxide to form calcium carbonate eventually.

Thus, the objective of this study is to explore a quick, easyto-implement, and efficient nanosurface modification method via a novel arc discharge plasma system to coat Ti samples with a nano thin film for improving MSC attachment, growth, and osteogenic differentiation. The principle method of nano deposition was employed through the use of cathodic vacuum arc discharge plasma. Cathodic vacuum arcs naturally produce a jet of ionized metallic vapor at hypersonic speeds through a voltage breakdown between two electrodes. Cathodic vacuum arcs have been used for a wide variety of applications such as thin films for semiconductive filaments, protective erosion and corrosion resistant films for cutting and forming tools, decorative coatings, jewelry, ${ }^{22-25}$ and now in our study for improved stem cell and orthopedic applications. For this purpose, we will utilize cathodic vacuum arc discharge plasma to deposit a thin, nanotextured film onto Ti samples to increase cell-Ti interaction. In this study, we focus on the effect of the plasma coating on surface morphology, hydrophilicity, specific proteins adsorption as well as MSC adhesion, proliferation, and osteogenic differentiation in vitro.

\section{Materials and methods Preparation of Ti samples}

Sheets of annealed Ti (95\% metals basis) foil with $0.5 \mathrm{~mm}$ thickness were obtained from Alfa Aesar (Ward Hill, MA, USA) and sectioned into $1 \mathrm{~cm} \times 1 \mathrm{~cm}$ squares using a vertical shear. The Ti squares were cleaned by being immersed in acetone, sonicated for 15 minutes, and rinsed three times with ultrapure water. Then the process was repeated with $70 \%$ ethanol and dried in an oven at $70^{\circ} \mathrm{C}$ overnight. The samples were then placed individually in a sterile 24-well plate before being exposed to arc discharge plasma (as described later) for $1-3$ seconds.

For this study, a cathodic arc jet was applied to create a thin film of Ti on small Ti samples. Experiments were conducted in a stainless steel vacuum chamber with dimensions of roughly $45 \mathrm{~cm}$ in diameter and $64 \mathrm{~cm}$ in length. The chamber was evacuated to a pressure of $10^{-4}$ Torr through the use of roughing and diffusion pumps. A photographic and schematic depiction of the chamber and experimental setup is shown in Figure 1. The tapered Ti cathode measured $\sim 6 \mathrm{~cm}$ in diameter at the base, $2.5 \mathrm{~cm}$ in depth, and with a $20^{\circ}$ taper. The cathode was connected to a welding power unit to provide $140 \mathrm{~A}$ and $35 \mathrm{~V}$ of arc current and voltage. The anode 


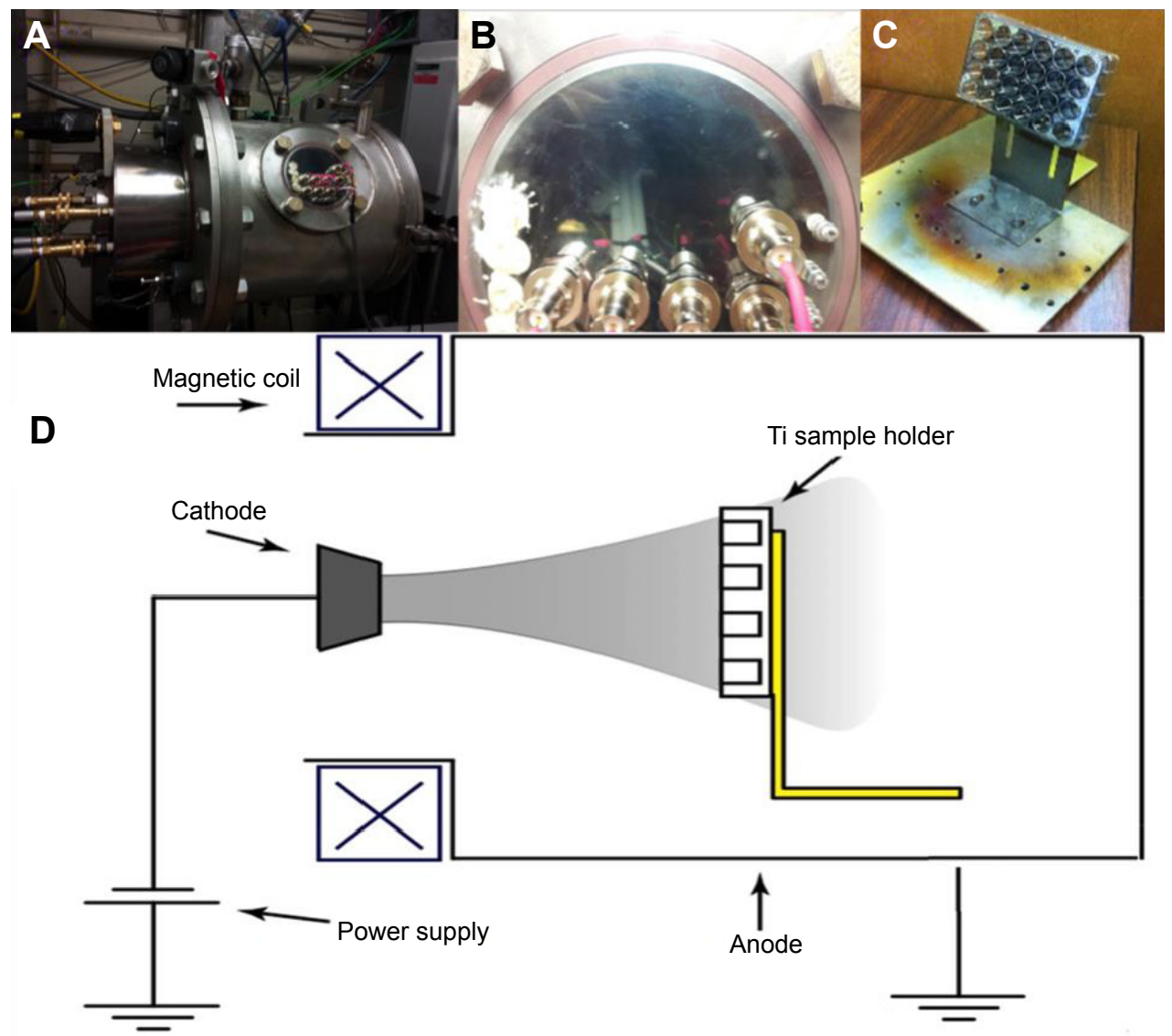

Figure I Photo images and schematic diagram of the cathodic arc discharge plasma generator used to treat the Ti and glass samples in this study.

Notes: (A and B) Photograph of the vacuum chamber within which plasma coating was deposited. (C) Photograph of mount used to orient well plates and allow for an even coating of samples. (D) Schematic diagram of the coating configuration used for samples in this study.

electrode was connected to a $10 \Omega$ current limiting resistor to the chamber walls and ground. A magnetic coil utilizing 11 A produced an axial magnetic field to the center of the cathode electrode in order to direct the plasma plume. The arc discharge was initiated through a physical contact triggering mechanism as the anode briefly touches the cathode, a voltage breakdown occurs between the two electrodes, generating a highly ionized metallic vapor plume. The Ti plasma generated through cathodic arc discharge is ionized to $\sim 99 \% .{ }^{26}$ Coated samples were reautoclaved prior to cell studies.

\section{Characterization of arc discharge plasma-coated $\mathrm{Ti}$}

A scanning electron microscopy (SEM, Zeiss NVision 40 FIB) equipped with an energy-dispersive X-ray spectrometer (EDS) was employed to qualitatively assess the surface morphologies and elements after nanocoating. Samples were imaged at $10 \mathrm{kV}$ at several magnifications. In addition, atomic force microscopy (AFM, Asylum, MFP-3D) was used to detect the surface roughness change after nanocoating.
Since the nanocoating cannot be imaged on the rough $\mathrm{Ti}$ foil, AFM samples were acquired by coating Ti plasma with 1 second and 2 seconds on cell culture polystyrene well plate, which could provide a very smooth surface. The thickness of coating was examined by detecting the interface between the coated and uncoated areas.

Contact angle analysis was performed on bare $\mathrm{Ti}$, and $\mathrm{Ti}$ samples treated for 1 second and 2 seconds with arc discharge plasma and analyzed via a drop shape analysis software (KRÜSS, Hamburg, Germany). This serves to indicate the hydrophilic character of tested samples. Specifically, $10 \mu \mathrm{L}$ drops were administered, and the placement and behavior of the drop were recorded by video camera at seven frames per second. The contact angle value was then calculated according to the spherical drop model from a single frame immediately after the drop was deposited. Measurements were reported as an average over four replicates.

Surface modification was also evaluated by adsorption of two specific proteins, fibronectin and vitronectin, via an enzyme-linked immunosorbent assay. In brief, samples were soaked in Dulbecco's Modified Eagle's Medium with 10\% 
fetal bovine serum for 24 hours at $37^{\circ} \mathrm{C}$. The samples were rinsed three times with phosphate-buffered saline (PBS) followed by blocking with $2 \%$ bovine serum albumin solution for 1 hour. After PBS washing, the first antibody (vitronectin antibody, 1:100 or fibronectin antibody, 1:100; Thermo Fisher Scientific, Waltham, MA, USA) was added to each sample and incubated for another 1 hour. Secondary antibody solution, goat anti-mouse immunoglobulin $(\mathrm{H}+\mathrm{L})(1: 100$ in $1 \%$ bovine serum albumin) was then added and incubated for 1 hour. Finally, 2,2'-azino-bis(3-ethylbenzothiazoline-6-sulfonic acid) (ABTS Substrate Kit; Vector Labs, Burlingame, CA, USA) was added to samples and incubated in the dark for 20 minutes at room temperature. Absorbance was analyzed at $405 \mathrm{~nm}$ by a spectrophotometer (Thermo Fisher Scientific).

\section{Human bone marrow MSC adhesion and proliferation study in vitro}

Primary human bone marrow MSCs were obtained from the Texas A\&M Health Science Center, Institute for Regenerative Medicine. They were cultured in standard MSC culture media consisting of alpha minimum essential medium (Thermo Fisher Scientific) supplemented with 16.5\% fetal bovine serum (Atlanta Biologicals, Lawrenceville, GA, USA), 1\% (v/v) L-glutamine (Thermo Fisher Scientific), and $1 \%$ penicillin:streptomycin (Thermo Fisher Scientific). MSCs of passages 3-6 were used for all of the in vitro testing.

For the MSC adhesion study, prepared Ti samples and glass coverslip references were placed into a 24-well plate, seeded with 20,000 cells $/ \mathrm{cm}^{2}$, and allowed to culture under standard cell culture conditions $\left(37^{\circ} \mathrm{C}\right.$, a humidified $5 \%$ $\mathrm{CO}_{2} / 95 \%$ air) for 4 hours. Nonadherent cells were removed, and adhered cells were counted via CellTiter $96^{\circledR}$ AQueous Non-Radioactive Cell Proliferation assay (MTS assay). Results are presented as absorbance after subtracting background. In addition, cell nuclei were stained and imaged using a laser scanning confocal microscope (Zeiss 710) to visualize the cell number difference among Ti and glass substrates with different coating times after 4 hours culture. Briefly, samples were washed twice with sterile PBS and fixed with $10 \%$ formalin for 15 minutes. Immediately following fixing, samples were washed twice with PBS and immersed in a solution of $0.1 \%$ Triton-X to permeabilize the cell membranes and allow for better dye infiltration. Samples were again washed twice with PBS and stained with 4',6-diamidino-2-phenylindole dihydrochloride (DAPI) for 10 minutes prior to imaging.

A 5 day MSC proliferation study was completed to evaluate stem cell growth behaviors with respect to plasma treatment times. The sample groups included untreated $\mathrm{Ti}$ control, untreated glass coverslip, Ti and glass samples exposed to 1-3 seconds of cathodic arc discharge plasma. Samples were placed into a 24-well plate and were seeded with 20,000 cells $/ \mathrm{cm}^{2}$. Seeded samples were cultured at standard cell culture conditions for 1 day, 3 days, or 5 days. Cell number was quantified using MTS assay at each time point. In order to visualize the cell spreading morphology on various Ti surfaces, confocal imaging was performed on $1 \mathrm{~cm}^{2}$ Ti samples that had been seeded with 20,000 cells and allowed to culture for 1 day, 3 days, and 5 days. Samples were prepared similarly to the aforementioned confocal imaged samples but with two additional PBS washes and staining with Rhodamine-Phalloidin for 20 minutes after DAPI staining. The double-stained samples were then imaged on a confocal microscope. Images presented here are mosaics of several images taken serially using the ZEN 2011 confocal controller software. All images were of a $1.1 \mathrm{~mm}^{2}$ representative section of a sample.

\section{MSC osteogenic differentiation}

MSCs were seeded at 100,000 cells $/ \mathrm{cm}^{2}$ on Ti control, Ti with 1 second and 2 seconds plasma treatment and cultured in osteogenic media (cell culture media supplemented with $10 \mathrm{nM}$ dexamethasone, $20 \mathrm{mM} \beta$-glycerolphosphate, and $50 \mu \mathrm{M}$ L-ascorbic acid 2-phosphate) for 1 week, 2 weeks, and 3 weeks. At each week, cells were lysed via three freeze-thaw cycles, and total calcium deposition, total protein production, and alkaline phosphatase (ALP) synthesis of MSCs were examined.

Specifically, total calcium deposition is one of the primary metrics used to determine the osteogenic character of bone marrow-derived MSCs. After the MSCs were lysed and removed, all substrates were immersed in a $0.6 \mathrm{~N} \mathrm{HCl}$ solution at $37^{\circ} \mathrm{C}$ for 24 hours. After the prescribed time period, the amount of dissolved calcium presented in the acidic supernatant was quantified via a commercial calcium assay (Pointe Scientific, Inc., Canton, MI, USA). A $50 \mu \mathrm{L}$ of sample solution was combined with the $o$-cresolphthalein complexone to form a purple tinted solution. The light absorbance was measured by a spectrophotometer at $570 \mathrm{~nm}$.

Total protein content of lysed samples was determined by a Micro BCA ${ }^{\mathrm{TM}}$ Protein Assay Kit (Thermo Fisher Scientific) according to the manufacturer's instruction. Specifically, $150 \mu \mathrm{L}$ of the cell lysate was loaded into a 96-well microplate. A $150 \mu \mathrm{L}$ of protein detection reagent was added and incubated for 2 hours at standard cell culture conditions. Absorbance of each sample was then read at $562 \mathrm{~nm}$ and compared against a standard curve derived 
from known concentrations of proteins to obtain total protein concentration of lysate aliquots.

ALP activity was also examined using a QuantiChrom ${ }^{\mathrm{TM}}$ Alkaline Phosphatase Assay Kit (BiopAssay Systems). A $50 \mu \mathrm{L}$ lysed solutions were transferred into 96-well plate followed by adding $150 \mu \mathrm{L}$ working solution to each well. The mixed solution was kept 4 minutes at room temperature to allow reaction. Absorbance was then read at $405 \mathrm{~nm}$. ALP activity was calculated according to the manufacturer's instruction.

\section{Statistical analysis}

All cellular experiments were run in triplicate and repeated three times. Data are presented as the mean value \pm standard error of the mean and were analyzed with student's $t$-test for pair-wise comparison. Statistical significance was considered at $P<0.05$.

\section{Results \\ Arc discharge plasma-coated Ti characterization}

This study evaluated the effects of arc discharge plasma coating on Ti samples for MSC response and osteogenic differentiation. Surface material characterization was performed first to allow for a better understanding of the effects of the type of coating used within this study. Utilizing only 1-2 seconds of exposure to arc discharge plasma, surface modification of Ti was evident as shown in Figure 2. Under low magnifications, there is no discerning difference among uncoated Ti control samples and the short duration
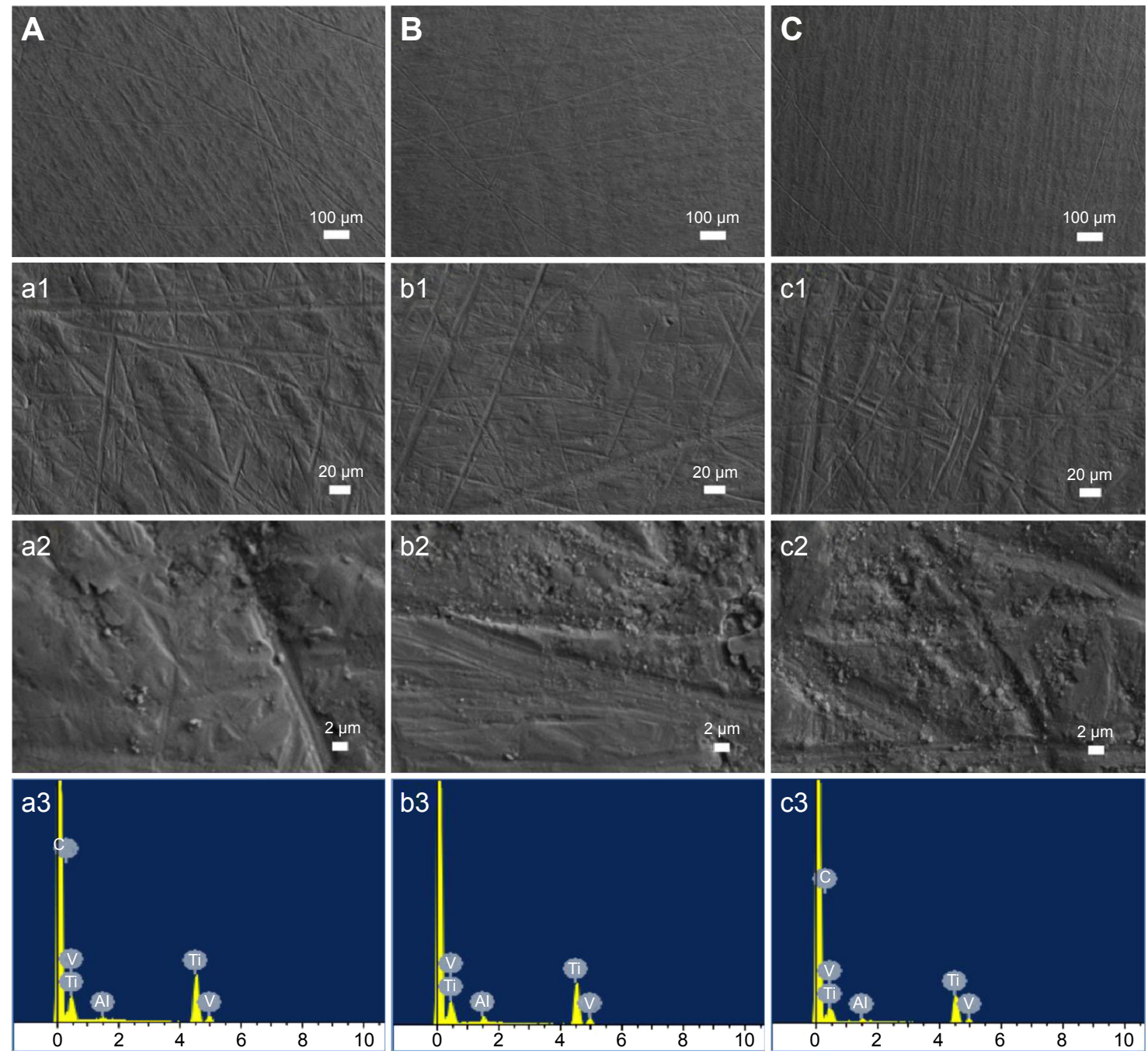

Figure 2 SEM morphologies of various substrates.

Notes: SEM images of Ti samples at low and high magnifications with various cathodic arc discharge plasma treatment times of I second (B, bl, and b2), and 2 seconds (C, cl, and c2) when compared to untreated Ti control (A, al, and a2). EDS pictures of elements on (a3) uncoated, (b3) I second nanocoated, and (c3) 2 seconds coated samples. Abbreviations: SEM, scanning electron microscopy; EDS, energy-dispersive X-ray spectrometer. 
plasma-delivered nanocoating samples. High magnification SEM images illustrate the nanoparticle deposition on the coated Ti samples as shown in Figure $2 \mathrm{~b} 2$ and $\mathrm{c} 2$. EDS evaluation indicates similar components for samples with and without coating, suggesting that the arc discharge plasma-delivered Ti nanoparticles on sample's surface. AFM examination showed the increase of surface roughness with coating time (Figure 3 ). Specially, 2 seconds plasma coating endowed a significant rougher surface than noncoated and 1 second coated samples (Table 1). The average thickness
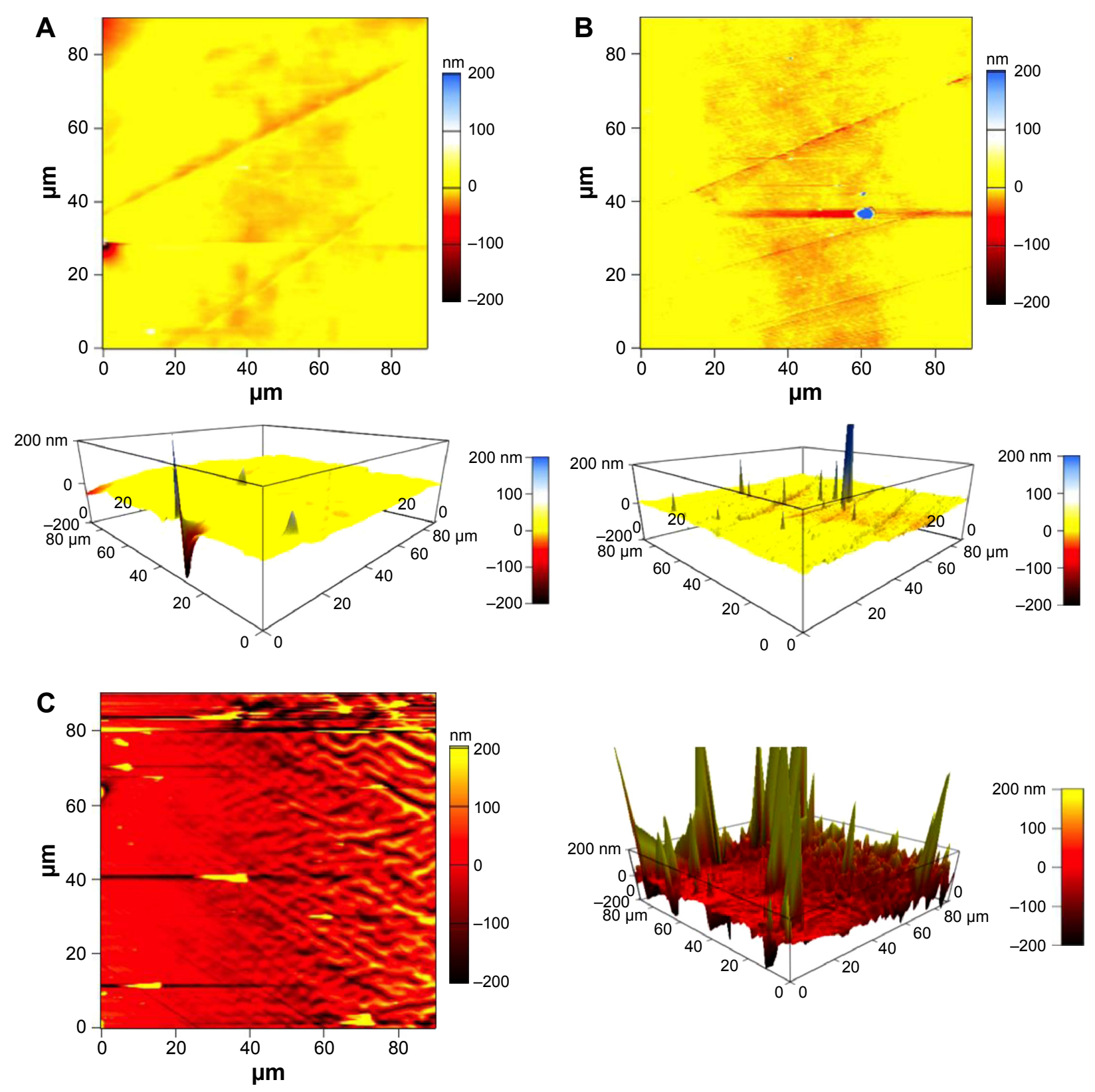

Figure 3 AFM images of samples with 0 seconds (A), I second (B), and 2 seconds (C) plasma coating. Abbreviation: AFM, atomic force microscopy.

of coating is $120 \mathrm{~nm}$ and $200 \mathrm{~nm}$ for 1 second and 2 seconds treated samples, respectively.

Besides the surface morphology and elements analysis, in order to investigate the underlying mechanisms of improved cell functions on nanocoated Ti samples (as shown in the "Greatly enhanced MSC adhesion and proliferation in vitro" and "MSC osteogenic differentiation in vitro" sections), the contact angle analysis and surface protein adsorption were conducted. Contact angle is a metric that can be used to elucidate the surface energy of the tested sample, and 
in turn, the relative hydrophobicity or hydrophilicity. The coated Ti samples were found to have a significantly lower contact angle than uncoated $\mathrm{Ti}$, indicating predominantly hydrophilic interactions at the surface (Figure 4). Similar to contact angle results, fibronectin adsorption was enhanced on the nanocoated surface (Figure 5A). There is no significant difference between the 1 second and 2 seconds coated groups, but they all outperformed the Ti controls. For the vitronectin, all groups showed similar adsorption level (Figure 5B).

\section{Greatly enhanced MSC adhesion and proliferation in vitro}

To further characterize the effect of the nanocoating on MSCs, a 4 hours MSC adhesion study was performed with both $\mathrm{Ti}$ and glass substrates (as references) as shown in Figure 6A. Uncoated Ti and uncoated glass were included as controls, and the results indicated significantly enhanced cell attachment on the plasma-coated samples, particularly, among the Ti samples. Ti samples with nanocoating significantly outperformed the Ti control without nanocoating. In particular, the Ti samples with 2 seconds of applied nanocoating performed better than all other sample groups and had a $327 \%$ increase in MSC adhesion when compared to the uncoated Ti controls. The Ti samples coated for 1 second and 3 seconds performed $80 \%$ and $148 \%$ better than the control group. This was further confirmed by confocal microscopy images that showed distinguishable cell density difference (Figure 6B). These results indicate that an ideal coating time may have been discovered. To further discern if an ideal coating time had been elucidated, a 5 days proliferation study was performed as seen in Figure 7. After 1 day, all samples treated with Ti nanocoating performed better than their uncoated controls. After 5 days, the highest cell densities were on the samples coated for 1 second and 2 seconds, which increased cell proliferation by $69 \%$ and $76 \%$, respectively. Glass references subjected to the same treatment exhibited a trend that was similar to the Ti.

Additionally, confocal microscopy imaging was performed for the outperformed 1-2 seconds coated samples

Table I Comparison of surface roughness alternation under different plasma-coating periods

\begin{tabular}{llll}
\hline & $\mathbf{0 ~ s}$ & I s & $\mathbf{2 ~ s}$ \\
\hline $\mathrm{Rq}(\mathrm{nm})$ & 9.6 & 17.4 & 89.5 \\
$\mathrm{Ra}(\mathrm{nm})$ & 6.6 & 9.2 & 50.2
\end{tabular}

Abbreviations: s, second; Rq, root mean square roughness of the surface; Ra, mean roughness of the surface.

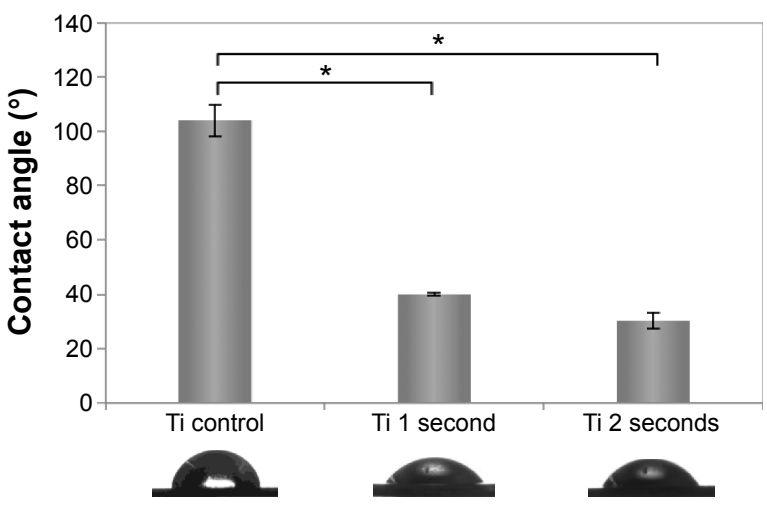

Figure $\mathbf{4}$ Increased surface hydrophilicity of $\mathrm{Ti}$ after exposure to I second and 2 seconds cathodic plasma treatment.

Notes: Data are mean \pm standard error of the mean, $n=4$. $* p<0.01$ when compared to Ti control. Bottom panels display the corresponding photographs of contact angle measurement.

with respect to uncoated control at 1 day, 3 days, and 5 days. Double-stained cell micrographs show that on plasma-coated samples, MSCs were more numerous and grouped closer together in a more confluent organization. Cells grown on coated samples exhibited a more flattened morphology with larger cell bodies and longer filopodia than cells grown on uncoated samples (Figure 8).

\section{MSC osteogenic differentiation in vitro}

A 3 weeks osteogenic differentiation study was performed to elucidate the potential advantage of nanocoated Ti samples over bare, uncoated Ti. As shown in Figure 9, calcium deposition dramatically increased in all groups at week 3 relative to 1 week and 2 weeks culture. Meanwhile, the lowest calcium deposition displayed on the uncoated group. Specifically, at week 3, cells cultured on the 1 second and 2 seconds nanocoated Ti samples had a $36.4 \%$ and $64.7 \%$ increase of calcium deposition when compared with that of uncoated Ti samples. Similar tendency was observed in total protein production (Figure 10). A significant increase was noted at week 3 for all groups. At week 1 and week 2, the nanocoated groups were still better than controls and this continued at week 3. ALP activity of MSCs cultured in osteogenic media at various times is shown in Figure 11. All ALP activity increased over time. Particularly, the ALP activity in the 1 second nanocoated group is higher than the uncoated control at week 1 . At week 2 and week 3, significantly higher ALP activity was detected in cells cultured on the 1 second and 2 seconds nanocoated groups when compared to uncoated controls.

\section{Discussion}

Ti orthopedic implants are durable and are able to withstand the intense forces orthopedic implants are necessarily subject 

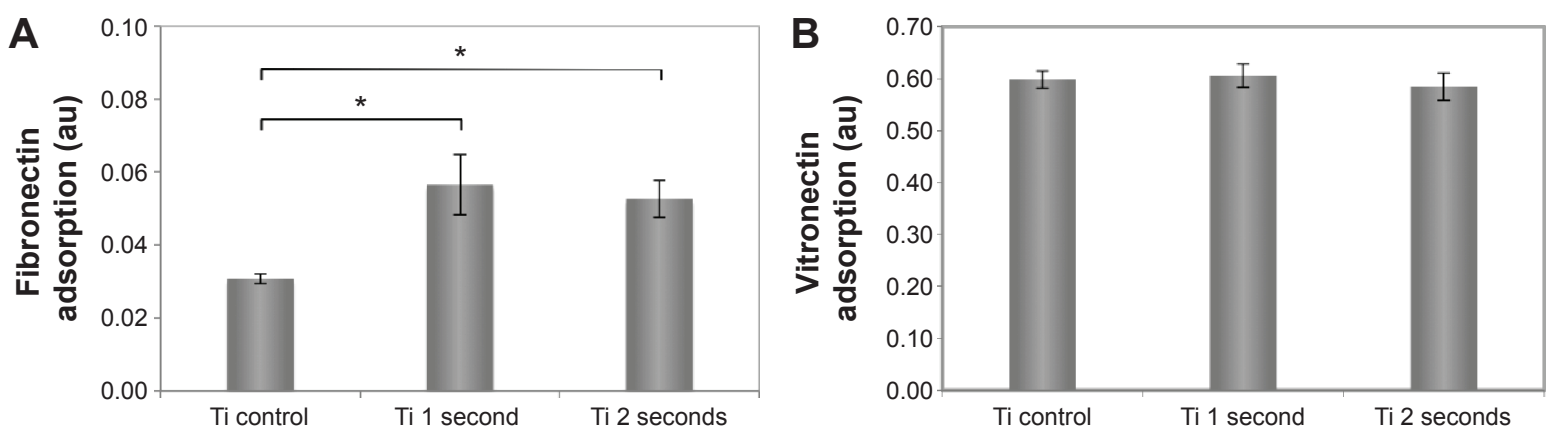

Figure 5 Fibronectin $(\mathbf{A})$ and vitronectin $(\mathbf{B})$ adsorption on Ti with I second and 2 seconds plasma coating compare to untreated group. Notes: $* \mathrm{p}<0.05$ when compared to Ti control. Data are mean \pm standard error of the mean, $\mathrm{n}=3$.

to. ${ }^{5}$ However, $\mathrm{Ti}$ is not considered as a highly bioactive material for ideal osseointegration as previously mentioned. To address this, many researchers are attempting to engineer bone substitutes, but there are few, if any, materials that can replace Ti in high-loading environments, and few that will be ready in the immediate future. To truly improve patient quality of life and reduce revision surgeries in the short term, Ti needs to be optimized. In addition, any improvements applied

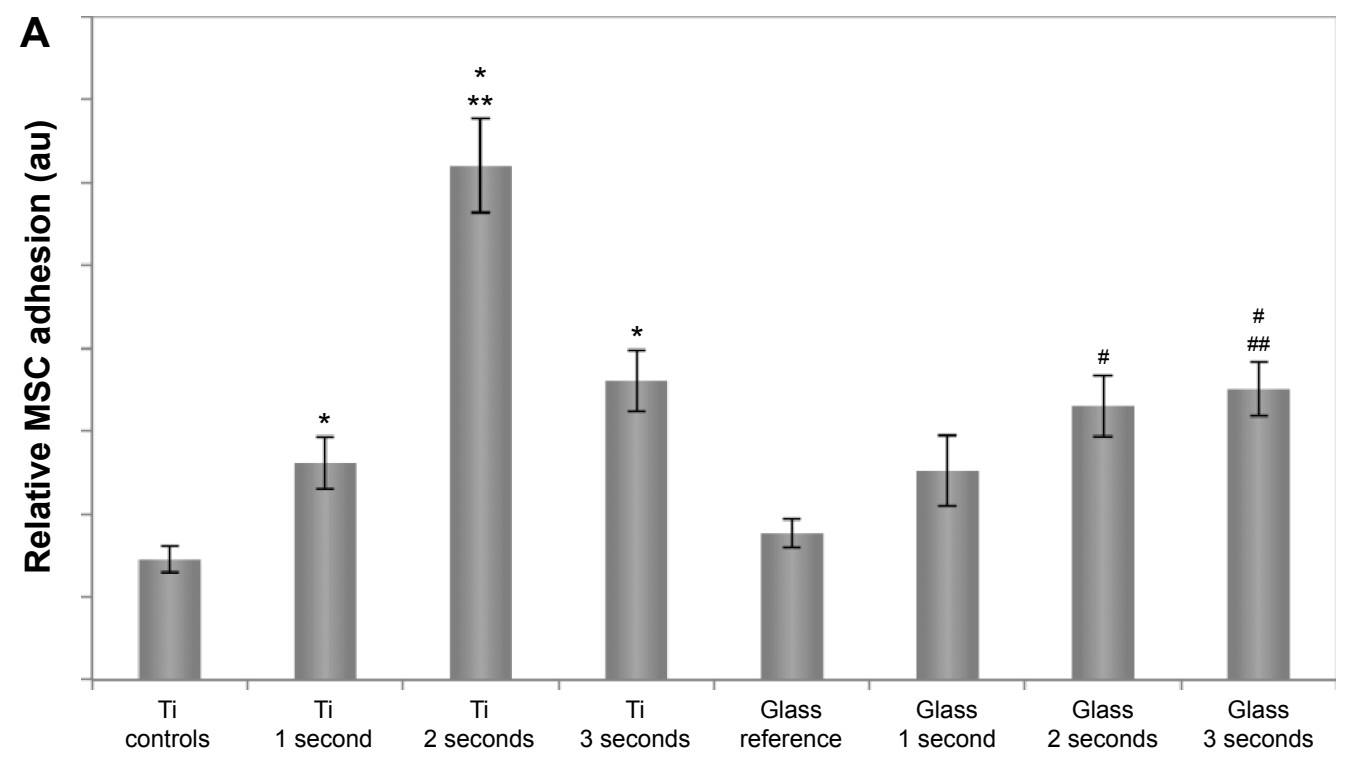

B

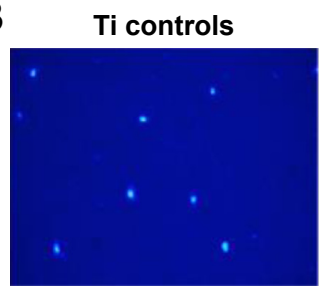

Glass reference

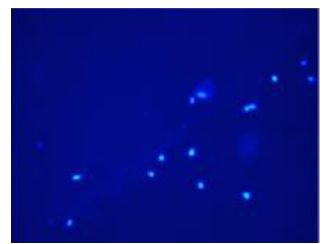

Ti 1 second

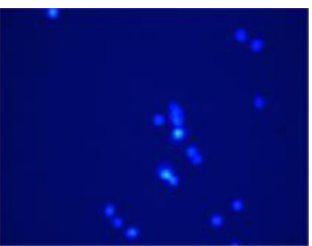

Glass 1 second

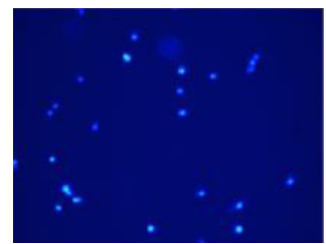

Ti 2 seconds

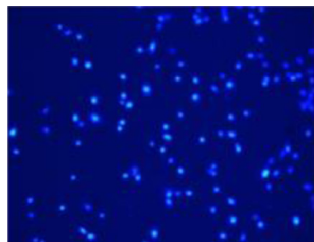

Glass 2 seconds

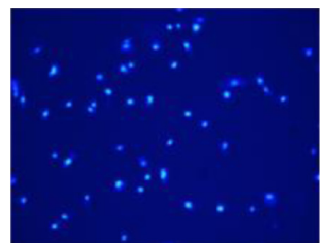

Ti 3 seconds

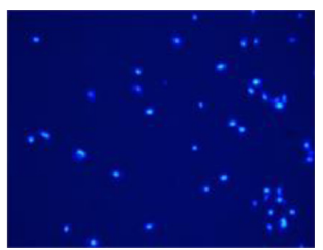

Glass 3 seconds

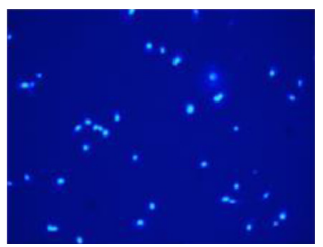

Figure 6 MSC adhesion on various substrates.

Notes: (A) Enhanced MSC adhesion on plasma-coated Ti after 4 hours of culture. Data are mean \pm standard error of the mean, $\mathrm{N}=3$. $* P<0.0 \mathrm{I}$ and $\# \boldsymbol{P}<0.0 \mathrm{I}$ when compared to respective Ti controls and glass references, ${ }^{* * P}<0.0 \mathrm{I}$ when compared to all other Ti samples, and ${ }^{\# P}<0.0 \mathrm{I}$ when compared to I second plasma-coated glass. (B) Fluorescence microscopy images of DAPI-stained MSC attachment density on various substrates (I0× magnification).

Abbreviations: MSC, mesenchymal stem cell; DAPI, 4',6-diamidino-2-phenylindole dihydrochloride. 


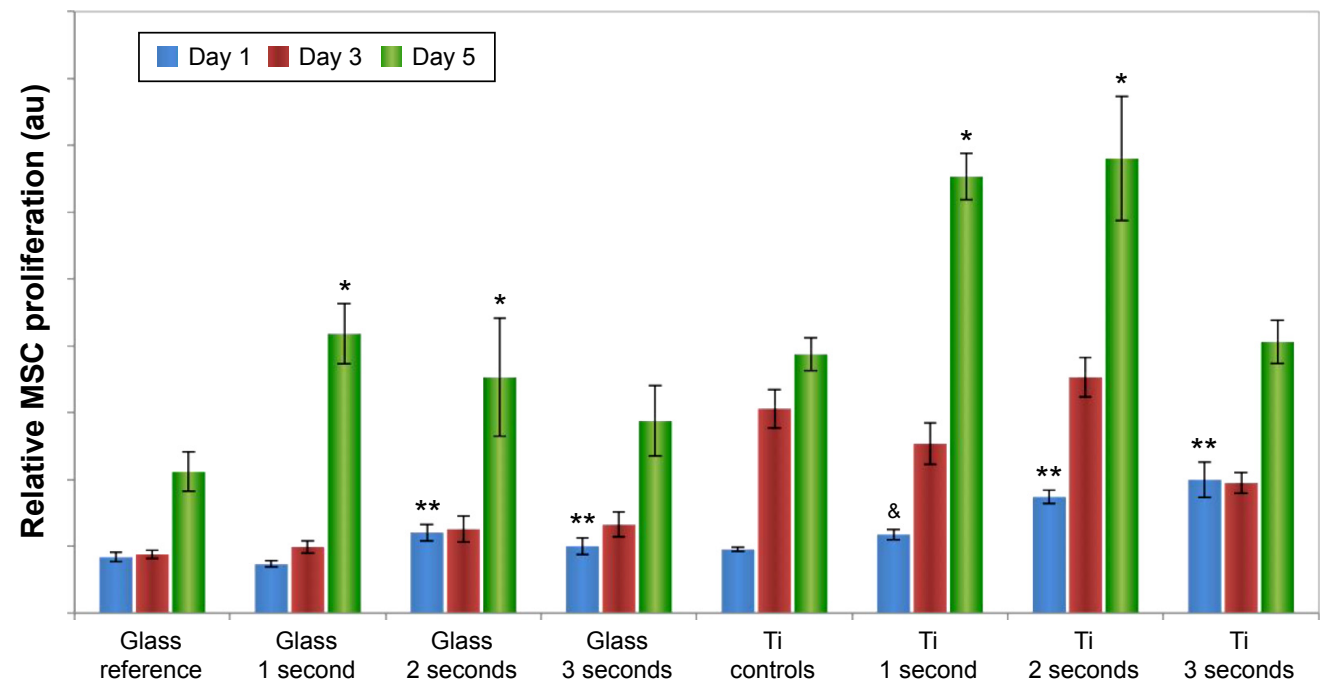

Figure 7 Significantly improved MSC proliferation on plasma-coated Ti.

Notes: Data are mean \pm standard error of the mean, $N=3$. $* P<0.05$ when compared to respective uncoated Ti controls and glasses after 5 days of culture, $* * P<0.05$ when compared to respective uncoated and I second plasma-coated $\mathrm{Ti}$ as well as glass after I day of culture, and $\& \mathrm{P}<0.05$ when compared to uncoated $\mathrm{Ti}$ controls. Abbreviation: MSC, mesenchymal stem cell.
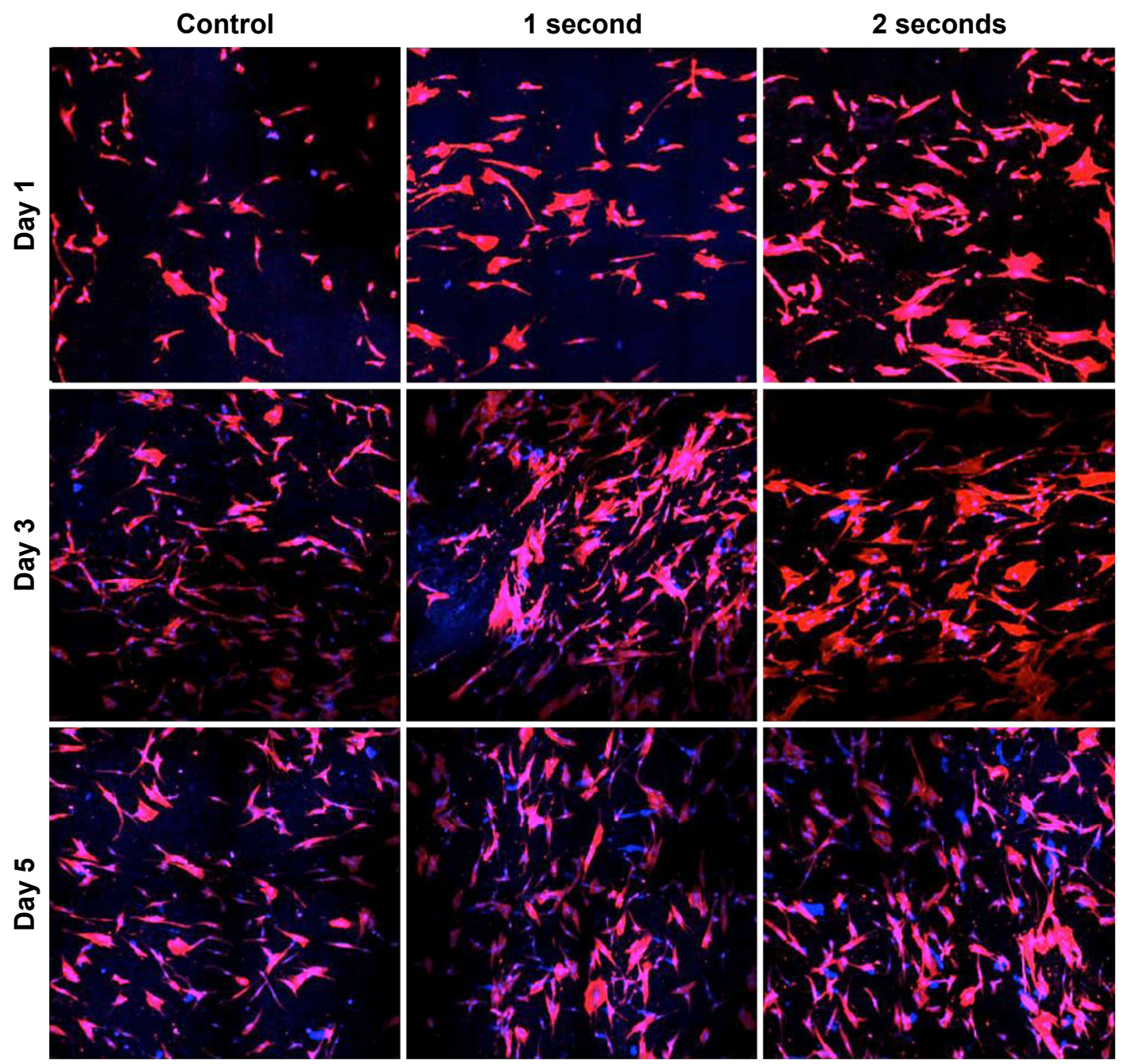

Figure 8 Typical MSC-spreading morphologies (confocal microscopy images, 20x magnification, images are $\sim \mathrm{mm}^{2}$ ) after I day, 3 days, and 5 days proliferation on: the Ti control, the I second plasma-coated $\mathrm{Ti}$, and the 2 seconds plasma-coated Ti samples.

Notes: Blue color represents nuclei stained by DAPI; red color represents cytoskeleton stained by Rhodamine-Phalloidin.

Abbreviations: MSC, mesenchymal stem cell; DAPI, 4',6-diamidino-2-phenylindole dihydrochloride. 


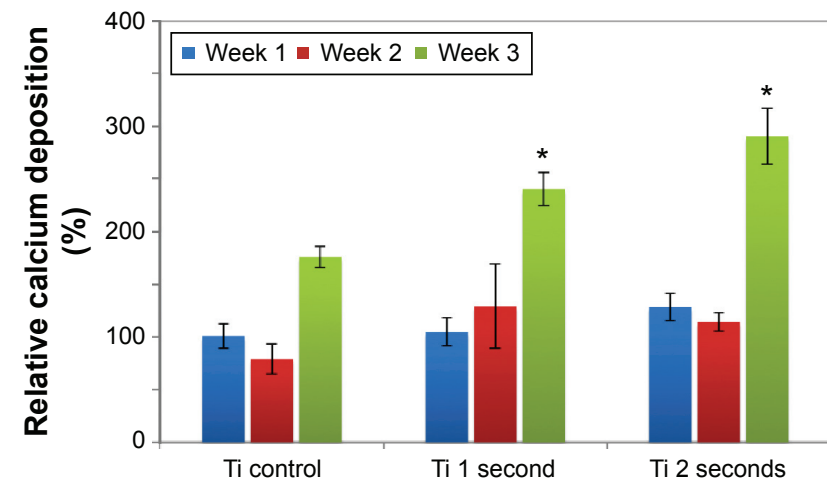

Figure 9 Enhanced total calcium deposition on plasma-coated Ti after 3 weeks of culture.

Notes: Data are mean \pm standard error of the mean, $\mathrm{N}=3$. $* \mathrm{P}<0.05$ when compared to Ti control at week 3 .

to Ti should be as simple as possible, both to reduce eventual cost of implementation and to minimize possible points of failure in the manufacturing process. If a simple method, such as the arc discharge coating method described herein, is comparable to existing technologies, the net result could be an improved Ti implant available to a greater number of people worldwide. As such, the objective of this study is to showcase the advantages of arc discharge plasma-coated $\mathrm{Ti}$ in improving MSC adhesion, proliferation, and calcium deposition, as well as the intrinsic surface properties modulated by the arc discharge plasma coating, markedly improved surface nanofeatures, contact angle, and protein adsorption.

As is evident from the SEM images in Figure 2, exposure to cathodic arc discharge plasma plume had some visible effects on surface morphology and nanotexturization of Ti. The uncoated Ti surface is distinguished by deep grooves from the manufacturer and cleaning processes. However, when

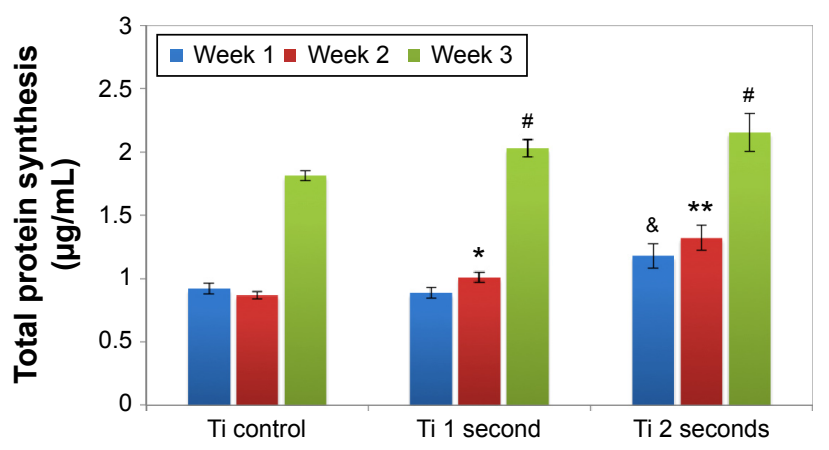

Figure 10 Total protein synthesis on various substrates after I week, 2 weeks, and 3 weeks of culture.

Notes: Plasma-coated $\mathrm{Ti}$ induced higher protein synthesis after 2 weeks and 3 weeks. Data are mean \pm standard error of the mean, $N=3$. ${ }^{\&} P<0.05$ when compared to all other samples at week $I, * P<0.05$ when compared to Ti control at week $2,{ }^{* * P}<0.05$ when compared to all other samples at week 2 , ${ }^{*} P<0.05$ when compared to Ti control samples at week 3 .

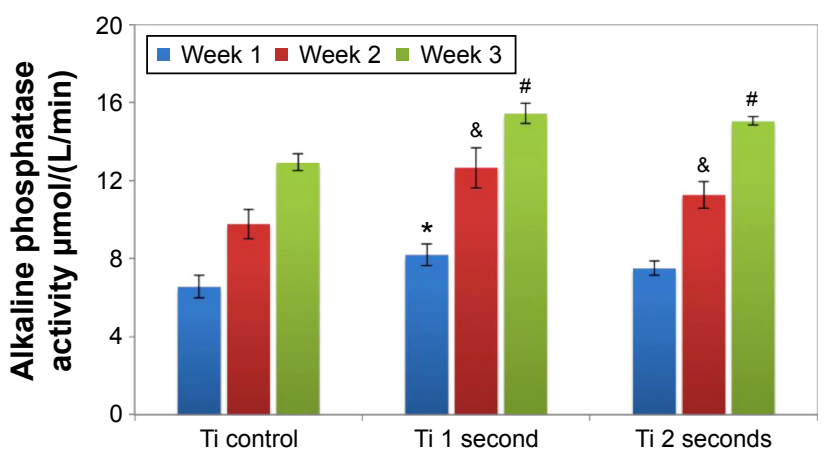

Figure I I Increased ALP activity on Ti with plasma coating.

Notes: Data are mean \pm standard error of the mean, $\mathrm{N}=3$. $* \mathrm{P}<0.05$ when compared to Ti control at week I, ${ }^{\&} P<0.05$ when compared to Ti control at week 2 , ${ }^{\#}<0.05$ when compared to Ti control samples at week 3.

Abbreviation: ALP, alkaline phosphatase.

observing a higher magnification of the macro grooves, the surface appears relatively smooth, with little or no nanotexturization. In contrast, the plasma-treated samples showed a different surface morphology at high magnification (Figure 2a2, $\mathrm{b} 2$, and c2). The control sample has many microsized grooves, but each groove is relatively smooth. The 1 second and 2 seconds coated samples presented some significant texture changes with more discernable nanoparticles from the control samples, which is confirmed by AFM analysis.

The texturization evident from the plasma nanocoating had a marked effect on the adhesion and proliferation of MSCs on the respective samples, likely due to the increase of available surface area and increased nanotexturization. Specifically, although the surface morphological change seems to apply rather linearly with time exposed to the plasma jet, MSC adhesion maximized on samples exposed to $\sim 2$ seconds of plasma exposure time. For proliferation study, 1 day, 3 days, and 5 days cultured MSC corroborated that 1 second and 2 seconds of plasma exposure was sufficient to elucidate a positive cellular response on treated Ti samples. Greatly increased cell numbers were observed on the Ti samples with 1-2 seconds plasma-deposited nanocoating after 5 days of culture. This large increase in proliferation indicates the positive effect of the nanocoating or additional topographical remodeling from the plasma jet on MSC growth. Beyond 3 seconds of plasma exposure, the Ti samples did not show a great increase in proliferation of MSCs after 5 days, the 4 hours adhesion even had a decrease when compared to 1 second and 2 seconds of exposure. It is possible that longer plasma treatment may result in more Ti particles deposition and aggregation, which can lead to a decrease of nanoroughness and a slightly lower cell adhesion and growth intensity. 
In addition, the plasma-deposited nanocoating not only modified the surface morphology of Ti samples but also contributed to a drastic decrease in contact angle at both evaluated treatment times. Contact angle is an important parameter in evaluating the surface wettability of a biomaterial construct, and as such, a lower contact angle can be attributed to improved MSC response. ${ }^{27,28}$ In this experiment, we observed a drastic decrease in contact angle between uncoated $\operatorname{Ti}\left(\sim 100^{\circ}\right)$ and coated $\operatorname{Ti}\left(\sim 30^{\circ}\right.$ and $40^{\circ}$ for 1 second and 2 seconds plasma treatment, respectively). The hydrophilic Ti surface after plasma coating may also contribute to the greatly improved MSC adhesion, proliferation, and differentiation as shown in our study. Meanwhile, improved fibronectin adsorption on the nanocoated surface might contribute to promoted cell's responses as well. It was documented that the surface fibronectin adsorption is directly related to cell functions. ${ }^{29}$

Moreover, our plasma Ti-coated samples required no chemical etchants or subsequent postprocessing. The treatment method uses a Ti plasma to modify the surface, thereby eliminating the possibility of metal contaminants. Additionally, it only requires clean Ti to be exposed to the plasma jet for 1 second or 2 seconds at most and requires no additional steps to arrive at the nanostructured surface. More importantly, Ti modified by arc discharge plasma nanocoating significantly improved osteogenic differentiation of MSCs as illustrated by calcium deposition, protein production, and ALP synthesis, therefore, is an alternative promising approach for the improved application of $\mathrm{Ti}$ in orthopedics.

\section{Conclusion}

In summary, this study has elucidated a new and simple surface modification method of increasing the hydrophilicity and nanosurface topography of Ti implants to improve MSC adhesion, proliferation, and osteogenic differentiation in vitro. We have shown that human bone marrow-derived MSCs displayed a significant increase in adhesion and proliferation on Ti substrates exposed to 1 second and 2 seconds arc discharge plasma coating, as evident by quantified cell number and through DAPI-stained fluorescent imaging. Confocal microscopy imaging of Ti samples over 1 day, 3 days, and 5 days of culture showed an increased degree of MSC spreading on Ti coated for 1 second and 2 seconds. More importantly, plasma-coated Ti samples were displaying increased calcium deposition, protein production, and ALP synthesis after 3 weeks culture of MSCs within osteogenic media when compared to uncoated Ti. The arc discharge plasma modification method requires no hazardous chemicals and can be completed in seconds as compared to more complex mechanical and chemical modification methods. Thus, it can be a promising single-step Ti surface modification method for improved orthopedic applications.

\section{Acknowledgments}

The authors would like to thank Research Award from the Clinical and Translational Science Institute at Children's National (CTSI-CN) and support from the George Washington Institute for Biomedical Engineering (GWIBE). The authors also would like to thank Sydney Butler and Hiwot Kassaye for their assistance with this project.

\section{Disclosure}

The authors report no conflicts of interest in this work.

\section{References}

1. Zhang L, Sirivisoot S, Balasundaram G, Webster TJ. Nanoengineering for bone tissue engineering. In: Khademhosseini A, Borenstein J, Toner M, Takayama S, editors. Micro and Nanoengineering of the Cell Microenvironment: Technologies and Applications. Norwood, MA: Artech House; 2008:431-460.

2. Steven K, Kevin O, Edmund L, Fionna M, Michael H. Projections of primary and revision hip and knee arthroplasty in the United States from 2005 to 2030. J Bone Joint Surg. 2007;89(4):780-785.

3. Rashid M, Stafford G, Chirodian N. Predicting the need for highdependency care in patients undergoing hip and knee revision surgery. Eur J Orthop Surg Traumatol. 2012;22(1):47-50.

4. Zhang L, Sirivisoot S, Balasundaram G, Webster TJ. Nanomaterials for improved orthopedic and bone tissue engineering applications. In: Basu B, Katti D, Kuma A, editors. Advanced Biomaterials: Fundamentals, Processing and Application. New York, NJ: John Wiley \& Sons Inc; 2009:205-241.

5. Gotfredsen K, Wennerberg A, Johansson C, Skovgaard LT, HjortingHansen E. Anchorage of TiO2-blasted, HA-coated, and machined implants: an experimental study with rabbits. J Biomed Mater Res. 1995; 29(10):1223-1231.

6. Goodheart JR, Miller MA, Mann KA. In vivo loss of cement-bone interlock reduces fixation strength in total knee arthroplasties. J Orthop Res. 2014;32(8):1052-1060.

7. Fennema P, Heyse TJ, Uyl-de Groot CA. Cost-effectiveness and clinical implications of advanced bearings in total knee arthroplasty: a long-term modeling analysis. Int J Technol Assess Health Care. 2014; 30:218-225.

8. Wang M, Castro NJ, Li J, Keidar M, Zhang LG. Greater osteoblast and mesenchymal stem cell adhesion and proliferation on titanium with hydrothermally treated nanocrystalline hydroxyapatite/magnetically treated carbon nanotubes. J Nanosci Nanotechnol. 2012;12(10): 7692-7702.

9. Zhang L, Webster TJ. Part III: nanotechnology for implants. In: Sattler K, editor. Handbook of Nanophysics: Nanomedicine and Nanorobotics. Boca Raton, FL: CRC Press; 2010:20-21.

10. Witek L, Marin C, Granato R, et al. Surface characterization, biomechanical, and histologic evaluation of alumina and bioactive resorbable blasting textured surfaces in titanium implant healing chambers: an experimental study in dogs. Int J Oral Maxillofac Implants. 2013; 28(3):694-700.

11. Prodanov L, Lamers E, Domanski M, Luttge R, Jansen JA, Walboomers XF. The effect of nanometric surface texture on bone contact to titanium implants in rabbit tibia. Biomaterials. 2013;34(12):2920-2927. 
12. Zhao $\mathrm{C}, \mathrm{Cao} \mathrm{P}, \mathrm{Ji} \mathrm{W}$, et al. Hierarchical titanium surface textures affect osteoblastic functions. J Biomed Mater Res A. 2011;99(4):666-675.

13. Yao C, Webster TJ. Anodization: a promising nano-modification technique of titanium implants for orthopedic applications. J Nanosci Nanotechnol. 2006;6(9-10):2682-2692.

14. Zhang L, Hemraz UD, Fenniri H, Webster TJ. Tuning cell adhesion on titanium with osteogenic rosette nanotubes. J Biomed Mater Res A. 2010;95(2):550-563.

15. Wennerberg A, Albrektsson T, Johansson C, Andersson B. Experimental study of turned and grit-blasted screw-shaped implants with special emphasis on effects of blasting material and surface topography. Biomaterials. 1996;17(1):15-22.

16. Buser D, Schenk RK, Steinemann S, Fiorellini JP, Fox CH, Stich H. Influence of surface characteristics on bone integration of titanium implants. A histomorphometric study in miniature pigs. J Biomed Mater Res. 1991;25(7):889-902.

17. Wall I, Donos N, Carlqvist K, Jones F, Brett P. Modified titanium surfaces promote accelerated osteogenic differentiation of mesenchymal stromal cells in vitro. Bone. 2009;45(1):17-26.

18. Wennerberg A, Jimbo R, Stubinger S, Obrecht M, Dard M, Berner S. Nanostructures and hydrophilicity influence osseointegration: a biomechanical study in the rabbit tibia. Clin Oral Implants Res. 2014; 25(9):1041-1050.

19. Chu PK. Plasma surface treatment of artificial orthopedic and cardiovascular biomaterials. Surf Coat Technol. 2007;201(9-11):5601-5606.

20. Chu PK, Chen JY, Wang LP, Huang N. Plasma-surface modification of biomaterials. Materi Sci Eng R. 2002;36(5-6):143-206.

21. Liu X, Poon RWY, Kwok SCH, Chu PK, Ding C. Structure and properties of Ca-plasma-implanted titanium. Surf Coat Technol. 2005; 191(1):43-48.
22. Boxman RL, Goldsmith S. Principles and applications of vacuum arc coatings. IEEE Trans Plasma Sci. 1989;17(5):705-712.

23. Boxman RL, Goldsmith S, Ben-Shalom A, et al. Filtered vacuum arc deposition of semiconductor thin films. IEEE Trans Plasma Sci. 1995; 23(6):939-944.

24. Keidar M, Beilis I, Boxman R, Goldsmith S. Macroparticle interaction with a substrate in cathodic vacuum arc deposition. Surf Coat Technol. 1996;86:415-420.

25. Keidar M, Beilis I. Plasma Engineering: Application in Aerospace, Nanotechnology and Bionanotechnology. 1st ed. Amsterdam, Netherlands: Elsevier; 2013.

26. Boxman RL, Sanders DM, Martin PJ. Books24x I. Handbook of Vacuum Arc Science and Technology: Fundamentals and Applications. Park Ridge, NJ: Noyes Publications; 1995.

27. Wang M, Cheng X, Zhu W, Holmes B, Keidar M, Zhang LG. Design of biomimetic and bioactive cold plasma-modified nanostructured scaffolds for enhanced osteogenic differentiation of bone marrow-derived mesenchymal stem cells. Tissue Eng Part A. 2014;20(5-6):1060-1071.

28. Rosales-Leal JI, Rodríguez-Valverde MA, Mazzaglia G, et al. Effect of roughness, wettability and morphology of engineered titanium surfaces on osteoblast-like cell adhesion. Colloid Surf A. 2010; 365(1-3):222-229.

29. Dolatshahi-Pirouz A, Jensen T, Kraft DC, et al. Fibronectin adsorption, cell adhesion, and proliferation on nanostructured tantalum surfaces. ACS Nano. 2010;4(5):2874-2882.
International Journal of Nanomedicine

\section{Publish your work in this journal}

The International Journal of Nanomedicine is an international, peerreviewed journal focusing on the application of nanotechnology in diagnostics, therapeutics, and drug delivery systems throughout the biomedical field. This journal is indexed on PubMed Central, MedLine, CAS, SciSearch ${ }^{\circledR}$, Current Contents ${ }^{\circledR} /$ Clinical Medicine,

\section{Dovepress}

Journal Citation Reports/Science Edition, EMBase, Scopus and the Elsevier Bibliographic databases. The manuscript management system is completely online and includes a very quick and fair peer-review system, which is all easy to use. Visit http://www.dovepress.com/ testimonials.php to read real quotes from published authors. 